\title{
Melioidosis in acute cholangitis of diabetic patient: a forgotten diagnosis
}

This article was published in the following Dove Press journal:

Research and Reports in Tropical Medicine

27 August 2012

Number of times this article has been viewed

\author{
Nasir Mohamad' \\ Suresh Ponnusamy ${ }^{2}$ \\ Sunita Devi ${ }^{3}$ \\ Rishya Manikam ${ }^{4}$ \\ Ilya Irinaz Idrus' \\ Nor Hidayah Abu Bakar ${ }^{5}$ \\ 'Department of Emergency Medicine, \\ School of Medical Sciences, Universiti \\ Sains Malaysia, Kubang Kerian, \\ Malaysia; ${ }^{2}$ AIMST University, Bedong, \\ Malaysia; ${ }^{3}$ Hospital Sultan Abdul Halim, \\ Sungai Petani, Malaysia; ${ }^{4}$ University \\ Malaya Medical Centre, Kuala Lumpur, \\ Malaysia; ${ }^{5}$ Department of Pathology, \\ Hospital Raja Perempuan Zainab II, \\ Kota Bharu, Malaysia
}

Correspondence: Nasir Mohamad Department of Emergency Medicine, School of Medical Sciences, Universiti Sains Malaysia, Health Campus, 16150 Kubang Kerian, Kelantan, Malaysia Tel +6097663219

Fax +6097663219

Email drnasirmohamadkb@yahoo.com
Abstract: Melioidosis presents with a wide range of clinical presentations, which include severe community-acquired pneumonia, septicemia, central nervous system infection, and less severe soft tissue infection. Hence, its diagnosis depends heavily on the clinical microbiology laboratory for culture. In this case report, we describe an atypical presentation of melioidosis in a 52-year-old man who had fever, right upper-abdominal pain, and jaundice for 15 days. Melioidosis caused by Burkholderia pseudomallei was subsequently diagnosed from blood culture. As a primary care physician, high suspicion index is of great importance. High suspicion index of melioidosis in a high-risk group patient, such as the patient with diabetes mellitus and diabetic foot, is crucial in view of atypical presentations of pseudomonas sepsis. A correct combination of antibiotic administration in the early phase of therapy will determine its successful outcome.

Keywords: Burkholderia pseudomallei, atypical, high suspicion, primary care

\section{Introduction}

Melioidosis is a bacterial disease caused by Burkholderia pseudomallei. It is endemic in most of the Southeast Asian countries including Malaysia. Although its true incidence and epidemiology in Malaysia is still unknown, in Alor Star alone (a city in the state of Kedah), the incidence of melioidosis is remarkably high at 16.35 per 100,000 population per year. ${ }^{1}$ In etiology, B. pseudomalleus is a Gramnegative aerobic bacillus. Due to its saprophytic nature, it is capable of surviving in any environment for years. ${ }^{2}$ Those conditions featuring qualitative and quantitative neutrophil abnormalities, such as alcoholism, diabetes mellitus, congestive heart failure, malignancy, and chronic renal failure, are recognized as risk factors for melioidosis. The percutaneous route, through an abraded or ulcerated skin, is thought to be the predominant route of entry, even for patients with pneumonic melioidosis. The wide ranges of clinical presentations include severe community-acquired pneumonia, septicemia, central nervous system infection, and less severe soft tissue infection. Diagnosis depends heavily on the clinical microbiology laboratory for culture. ${ }^{3}$ Although a wide variety of rare presentations are reported for melioidosis, acute cholangitis has previously been reported only once. ${ }^{4}$ In this case report, we would like to share the atypical presentation of melioidosis in a 52-year-old man who had fever, right upper-abdominal pain, and jaundice for 15 days. A diagnosis of melioidosis was subsequently diagnosed, after the blood culture confirmed the presence of B. pseudomallei. 


\section{Case report}

A 52 year-old male patient, who was a known to have type 2 diabetes mellitus for the past 18 years, presented to hospital with a fever of 15 days. This was a high-grade fever, intermittent in duration, associated with chills and rigor. It was also associated with vomiting, and right upper-quadrant pain. He had productive cough with scanty mucoid sputum for duration of 1 week that was associated with difficulty in breathing. He denied coughing up blood. No history of dysuria or loin pain was reported. His urine was dark in color.

The patient was continued on previous treatment with gliclazide tablets $80 \mathrm{mg}$ twice daily, and aspirin tablets $75 \mathrm{mg}$ once daily. He had also previously (prior to admission) been started on rifampicin capsules $450 \mathrm{mg}$ once daily, and fusidic acid tablets $250 \mathrm{mg}$ twice a day, for methicillin-resistant Staphylococcus aureus (MRSA) (which was cultured in his right foot ulcer) for 14 days. There was no history of jaundice in the past.

After admission, he was started on insulin for diabetic control. Viral markers for hepatitis and ELISA for human immunodeficiency virus were found to be negative.

General examination findings were that the patient was febrile, pale and deeply icteric, but not toxic. His pulse rate, blood pressure and respiratory rate were within normal limits, and jugular venous pressure was not elevated. Apart from these, there were no abnormal findings on general examination.

On systemic examination, respiratory and cardiovascular systems were normal. Severe tenderness was noted in the right-upper quadrant of the patient's abdomen, yet Murphy's sign was negative. There was no clinical evidence of hepatosplenomegaly.

Examination of the patient's right foot revealed that a $4 \times 4 \mathrm{~cm}$ ulcer was present in the sole of the foot, which was dry, clean, and looked healthy. He had been taking rifampicin capsules and fusidic acid tablets, as previously MRSA was grown in the wound swab.

Laboratory investigation results are summarized in Table 1.

Ultrasound of the patient's abdomen showed that the liver appeared homogenous, with no focal lesion. No intrahepatic biliary tree dilatation was noted. The gall bladder was distended but without calculi. The common bile duct was $7 \mathrm{~mm}$ in size. The pancreas was not visualized due to bowel gas. Both kidneys were normal in size and echo texture. The urinary bladder was well distended. Minimal free fluid was present.

Chest X-ray revealed right upper-lobe consolidation, and the patient was started on azithromycin $500 \mathrm{mg}$ once daily
Table I Investigation results

\begin{tabular}{llll}
\hline No & Investigation & Value & (Normal value) \\
\hline $\mathrm{I}$ & Total white blood cell count & $17.4 \times 10^{9} / \mathrm{L}$ & $3.8-9.7 \times 10^{9} / \mathrm{L}$ \\
2 & Platelet & $251 \times 10^{9} / \mathrm{L}$ & $167-376 \times 10^{9} / \mathrm{L}$ \\
3 & ALT & $155 \mu / \mathrm{L}$ & $\leq 40 \mu / \mathrm{L}$ \\
4 & AST & $124 \mu / \mathrm{L}$ & $\leq 37 \mu / \mathrm{L}$ \\
5 & ALP & $1022 \mu / \mathrm{L}$ & $39-117 \mu / \mathrm{L}$ \\
6 & Total bilirubin & $343 \mu \mathrm{mol} / \mathrm{L}$ & $3.4-17.1 \mu \mathrm{mol} / \mathrm{L}$ \\
7 & Direct bilirubin & $242 \mu \mathrm{mol} / \mathrm{L}$ & $0.8-5.1 \mu \mathrm{mol} / \mathrm{L}$ \\
8 & Indirect bilirubin & $101 \mu \mathrm{mol} / \mathrm{L}$ & $2.6-12.0 \mu \mathrm{mol} / \mathrm{L}$ \\
9 & Serum amylase & $118 \mu / \mathrm{L}$ & $\leq 200 \mu / \mathrm{L}$ \\
15 & Urine bilirubin & $3+(12 \mathrm{mg} / \mathrm{dL})$ & Negative $(-)$ \\
16 & Urine urobilinogen & $3+(12 \mathrm{mg} / \mathrm{dL})$ & $1-2 \mathrm{mg} / \mathrm{dL}$ \\
19 & Blood smear for & Negative & Negative \\
& malaria parasite & & \\
\hline
\end{tabular}

Abbreviations: ALT, alanine aminotransferase; ALP, alkaline aminotransferase; AST, aspartate aminotransferase.

along with gentamycin $80 \mathrm{mg}$ twice daily, for a respiratory tract infection. The sputum culture did not show any growth of pathogenic organism. The sputum smear for acid-fast bacillus was also found to be negative in three specimens.

The patient was started on ceftazidime by injection $2 \mathrm{~g}$ three times a day empirically for 4 weeks, after sending blood specimens for culture and sensitivity. After 7 days, blood culture and sensitivity test revealed B. pseudomallei, which were sensitive to ceftazidime, imipenem, and piperazillin/ tozabactum.

After 1 week, his fever subsided. The right hypochondriac pain improved and was further referred to the surgical team. Patient's glycemic status was managed with insulin injection. After a 28-day course of parenteral antibiotic, his liver enzymes normalized, and he was started on doxycycline and trimethoprim-sulfamethoxazole, which he was advised to continue for another 6 months.

\section{Discussion}

On initial assessment, our impression was acute cholangitis in view of the given history of high-grade fever, jaundice, and right hypochondriac pain. However, ultrasonography of the hepatobiliary system did not suggest the presence of calculus or acalculous cholecystitis, except for a distended gall bladder. The liver architecture was normal. Other differential diagnoses were community-acquired pneumonia, and acute viral hepatitis.

Laboratory investigations revealed a condition predominantly cholestatic icterus in origin, evidenced by marked elevation of alkaline phosphatase (Table 1).

In this case, our managing team decided to start with intravenous ceftazidime $2 \mathrm{~g}$ three times a day empirically 
in view of a considered complicated infection, based on the premorbid history of diabetes mellitus and focal foot ulcer (the probable causative root of infection for pseudomonas infection complicated with sepsis). Eventually, blood culture revealed the growth of $B$. pseudomallei, which was sensitive to ceftazidime. Thus, the patient recovered well thereafter.

Burkholderia pseudomalleus is a Gram-negative aerobic bacillus, which by its saprophytic nature is capable of surviving in any environment for years. ${ }^{2}$ Those conditions featuring qualitative and quantitative neutrophil abnormalities, such as alcoholism, diabetes mellitus, congestive heart failure, malignancy, and chronic renal failure, are recognized as risk factors for melioidosis. The percutaneous route, through an abraded or ulcerated skin, is thought to be the predominant route of entry, even for patients with pneumonic melioidosis.

The clinical manifestations of melioidosis are protean and may involve virtually every organ in the body, though pneumonia and septicemia are the much more common forms. A substantial percentage of melioidosis cases manifest with bacteremia (40\%-60\%), septic shock (20\%), and mortality is high (up to 60\%). ${ }^{5}$ Pneumonia is by far the most common presentation of $B$. pseudomallei infection, occurring in half the cases. Pneumonia in melioidosis may range in severity and may be acute, subacute, or chronic, with the chronic type resembling tuberculosis. A wide variety of other problems may occur, which usually require surgery, including prostatic abscesses, paralytic encephalomyelitis, or intra-abdominal abscess formation. ${ }^{6}$ Mortality rates among patients with bacteremia and multiorgan involvement, or septic shock may be as high as $90 \% .^{7}$

Owing to their ability to survive in any environment, standard specimen collection and transport principles are sufficient for recovering Burkholderia species in clinical practice. It can be cultured from sputum, blood, and other clinical samples. The organism grows more slowly than other bacteria that may be present in clinical specimens, and in specimens from nonsterile sites, is easily overgrown. Nonsterile specimens, especially sputum, should therefore be cultured in selective media such as modified Ashdown $\operatorname{agar}^{8}$ or B. cepacia medium. Its large wrinkled colonies look like environmental contaminants and are therefore often discarded as being of no clinical significance. Even when the isolate is recognized to be significant, commonly used identification systems may misidentify the organism as Chromobacterium violaceum or other nonfermenting Gram-negative bacilli, such as B. cepacia or Pseudomonas aeruginosa. Serological tests, such as indirect hemagglutination assay, can help in making a diagnosis of melioidosis, but the sensitivity and specificity is limited (71.4\% and $63.7 \%$, respectively). ${ }^{9}$

Cochrane Collaboration review ${ }^{10}$ recommends intravenous therapy for acute melioidosis using a third generation cephalosporin, such as ceftazidime, or imipenem (or meropenem) as a first line agent, or a beta-lactam/betalactamase inhibitor combination (amoxicillin-clavulanate) as second-line treatment. Initial-phase therapy should last at least 14 days, while 6 weeks of intravenous therapy is indicated for suppurative complications (eg, intra-abdominal abscesses or septic arthritis). For the eradication phase, oral therapy should include a combination of trimethoprim-sulfamethoxazole and doxycycline ${ }^{11}$ for duration of at least 3 to 6 months. Oral monotherapy with trimethoprim-sulfamethoxazole or amoxicillin-clavulanate (in pregnant women or children) is also acceptable.

This patient had a diabetic foot ulcer, which was likely to have been the portal of entry for the organism. ${ }^{12}$ Diabetes was the important predisposing factor for melioidosis in this patient. The likely reason for severe obstructive jaundice in this patient was acute cholangitis, as evidenced by fever, right upper-quadrant pain, and jaundice, and mild dilatation of the common bile duct as observed by abdominal ultrasound. Rifampicin may cause elevation of liver enzymes and cholestatic picture when used with fusidic acid, as was seen in this patient (Table 1). Other causes of obstructive jaundice in this patient were ruled out by relevant investigations.

Adequate patient history supplemented with proper physical examination is pertinent in diagnosis making, and both should be the mainstay of clinical approach. If such an approach is undertaken, empirical antibiotic aimed at eliminating $B$. pseudomallei can be administered earlier in the treatment and management course, rather than simply waiting for the culture result, by which the time the patient's condition may have deteriorated greatly.

\section{Conclusion}

As a primary care physician, high suspicion index is of great importance. High suspicion index of melioidosis in a highrisk group patient, such as the patient with diabetes mellitus and diabetic foot, is crucial in view of atypical presentations of pseudomonas sepsis. A correct combination of antibiotic administration in the early phase of therapy will determine its successful outcome.

\section{Disclosure}

The authors report no conflicts of interest in this work. 


\section{References}

1. Hassan MR, Pani SP, Peng NP, et al. Incidence, risk factors and clinical epidemiology of melioidosis: a complex socio-ecological emerging infectious disease in the Alor Setar region of Kedah, Malaysia. BMC Infect Dis. 2010;10:302.

2. Chierakul W, Winothai W, Wattanawaitunechai C, et al. Melioidosis in 6 tsunami survivors in southern Thailand. Clin Infect Dis. 2005;41(7):982-990.

3. Inglis TJ, Rolim DB, Rodriguez JL. Clinical guideline for diagnosis and management of melioidosis. Rev Inst Med Trop Sao Paulo. 2006;48(1):1-4.

4. Lai CH, Huang CK, Chin C, et al. Acute septicemic melioidosis presenting with acute cholangitis. Infection. 2007;35(6):461-464.

5. Currie BJ, Fisher DA, Howard DM, et al. Endemic melioidosis in tropical northern Australia: a 10-year prospective study and review of the literature. Clin Infect Dis. 2000;31(4):981-986.

6. Dance DA, Wuthiekanun V, Naigowit P, White NJ. Identification of Pseudomonas pseudomallei in clinical practice: use of simple screening tests and API 20NE. J Clin Pathol. 1989;42(6):645-648.
7. Currie BJ, Fisher DA, Howard DM, et al. The epidemiology of melioidosis in Australia and Papua New Guinea. Acta Trop. 2000;74(2-3): 121-127.

8. Currie BJ. Burkholderia pseudomallei and Burkholderia mallei: melioidosis and glanders. In: Mandell GL, Bennett JE, Dolin R, editors. Principles and Practice of Infectious Diseases. 6th ed. New York, NY: Churchill Livingstone; 2005:2622-2632.

9. Limmathurotsakul D, Jamsen K, Arayawichanont A, et al. Defining the true sensitivity of culture for the diagnosis of melioidosis using Bayesian latent class models. PLoS ONE. 2010;5(8):e12485.

10. Samuel M, Ti TY. Interventions for treating melioidosis. Cochrane Database Syst Rev. 2001;2:CD001263.

11. Chaowagul W, Suputtamongkol Y, Dance DA, Rajchanuvong A, Pattara-arechachai J, White NJ. Relapse in melioidosis: incidence and risk factors. J Infect Dis. 1993;168(5):1181-1185.

12. Northern Territory Government Department of Health. Melioidosis Fact Sheet. Centre for Disease Control; Dec 2009. Available from http:// www.nt.gov.au/health. Accessed August 6, 2012.

\section{Publish your work in this journal}

Research and Reports in Tropical Medicine is an international, peerreviewed, open access journal publishing original research, case reports, editorials, reviews and commentaries on all areas of tropical medicine, including: Diseases and medicine in tropical regions; Entomology; Epidemiology; Health economics issues; Infectious disease; Laboratory science and new technology in tropical medicine; Parasitology; Public health medicine/health care policy in tropical regions; and Microbiology. The manuscript management system is completely online and includes a very quick and fair peer-review system. Visit http://www.dovepress. com/testimonials.php to read real quotes from published authors. 